\title{
ESTADO HÍDRICO Y CAMBIOS ANATÓMICOS EN LA CALABACITA (Cucurbita pepo L.) ALMACENADA
}

\author{
WATER STATUS AND ANATOMIC CHANGES IN STORED \\ ZUCCHINI (Cucurbita pepo L.)
}

\author{
Vania Urías Orona ${ }^{1}$, Dolores Muy Rangel ${ }^{1 *}$, Tomás Osuna Enciso ${ }^{1}$, Adriana Sañudo Barajas ${ }^{1}$, \\ Manuel Báez Sañudo ${ }^{1}$, Benigno Valdez Torres ${ }^{1}$, Jorge Siller Cepeda ${ }^{1}$ y Juan Campos Sauceda ${ }^{2}$
}

\begin{abstract}
${ }^{1}$ Centro de Investigación en Alimentación y Desarrollo, A. C., Coordinación Culiacán. Carr. Eldorado Km.5.5, Campo el Diez. 80110, Culiacán, Sinaloa, México. ${ }^{2}$ Instituto Tecnológico de Culiacán. Juan de Dios Bátiz No. 310 pte., Col. Guadalupe. 80220, Culiacán, Sinaloa, México
\end{abstract}

*Autor para correspondencia (mdmuy@ciad.edu.mx)

\section{RESUMEN}

El fruto de calabacita (Cucurbita pepo L.) es una hortaliza que presenta elevada actividad metabólica, alta transpiración y sensibilidad a daños mecánicos en postcosecha. En este estudio se evaluó el efecto de la temperatura de almacenamiento en el estado hídrico, la integridad celular y la calidad postcosecha de frutos de calabacita tipo Zucchini cv. 'Raben'. Frutos cosechados en madurez hortícola se almacenaron por 12 d a 10 y $20{ }^{\circ} \mathrm{C}$, y 85 a $87 \%$ de humedad relativa. Se evaluó pérdida de peso, marchitamiento, contenido relativo de agua (CRA), potencial hídrico $\left(\Psi_{w}\right)$, potencial osmótico $\left(\Psi_{s}\right)$, potencial de presión $\left(\Psi_{p}\right)$ e integridad celular mediante un estudio histológico de los frutos. Los primeros síntomas de marchitez del fruto aparecieron cuando hubo $6 \%$ de pérdida de peso. La plasmólisis celular se presentó entre 81 y $82 \%$ de $C R A$, con un $\Psi_{p}$ igual a cero y con $\Psi_{w}$ y $\Psi_{s}$ iguales a $-0.8 \mathrm{MPa}$. La integridad del tejido se afectó por la pérdida de adhesión de las células de la zona del endocarpio, que luego progresó hacia el epicarpio del fruto.

Palabras claves: Cucurbita pepo, almacenamiento, pérdida de peso, potencial hídrico, plasmólisis.

\section{SUMMARY}

Zucchini fruits (Cucurbita pepo L.) show high metabolic activity and transpiration rates, and are highly susceptible to mechanical damage during harvest and postharvest. In this study, the effect of storage temperature on water status, cellular integrity and postharvest quality of zucchini were evaluated. Fruits in horticultural maturity stage $s$ were stored $12 \mathrm{~d}$ at 10 and $20^{\circ} \mathrm{C}$, and 85 to $87 \%$ relative humidity. Weight loss, relative water content $(R W C)$, water potential $\left(\Psi_{w}\right)$, osmotic potential $\left(\Psi_{\pi}\right)$, pressure potential $\left(\Psi_{p}\right)$, and cellular integrity observed through histological studies in fruits, were analyzed. The first sign of wilting ocurred with $6 \%$ of weight loss. Cell tissue plasmolysis occurred between 81 and $82 \%$ of $R W C$, when $\Psi_{p}$ was equal to zero and $\Psi_{w}$ and $\Psi_{\pi}$ were equal to - $0.8 \mathrm{MPa}$. Fruit tissue integrity was affected by the loss of adhesion cells at the endocarp zone which progressed to the pericarp.

Index words: Cucurbita pepo, storage, weight loss, water potential, plasmolysis.

\section{INTRODUCCIÓN}

México es uno de los principales productores de calabaza (Cucurbita pepo L.) a nivel mundial. La producción nacional en 2010 fue de 444520 t, de las cuales el Estado de Sinaloa fue el principal productor con $18 \%$ (SIAP, 2012). El fruto de calabacita Zucchini (C. pepo zucchini) se cosecha inmaduro en un estado temprano de crecimiento (Carvajal et al., 2011) y con un escaso desarrollo de cutícula, lo cual facilita el daño por cortes y abrasiones, una transpiración elevada y en general, la pérdida de calidad durante el manejo postcosecha (Mohammed y Brecht, 2003; Brew et al., 2006). Fisiológicamente este fruto presenta un comportamiento no climatérico con producción de $\mathrm{CO}_{2}$ moderada de 17 a 18 y de 42 a $48 \mathrm{~mL} \mathrm{~kg}^{-1} \mathrm{~h}^{-1}$, a 10 y $20^{\circ} \mathrm{C}$, respectivamente (Suslow y Cantwell, 2009). Las condiciones de temperatura de almacenamiento inferiores o superiores a las recomendadas $\left(5\right.$ a $10{ }^{\circ} \mathrm{C}$ y 90 a $95 \%$ de HR), afectan la calidad y vida postcosecha de los frutos de Zucchini (Carvajal et al., 2011).

La exposición del fruto a temperaturas inferiores a 5 ${ }^{\circ} \mathrm{C}$ por 2 a $3 \mathrm{~d}$ inducen daño por frío y la pérdida total de calidad (Balandrán-Quintana et al., 2003; Carvajal et al., 2011). El deterioro de la calidad comercial de las cucurbitáceas se manifiesta por la pérdida de firmeza y por la aparición de signos de marchitamiento, ambos eventos asociados con cambios en el estado hídrico del tejido cuyo umbral de pérdida de agua es de $6 \%$ (Sargent y Maynard, 2002; Brew et al., 2006). El almacenamiento a temperatura y déficit de presión de vapor elevado y a humedad relativa baja, aceleran el deterioro de los frutos y causan plasmólisis celular (Paull, 1999; Muy et al., 2004). En calabacita se busca conocer si la pérdida de calidad ocasionada por el marchitamiento se relaciona con cambios en la integridad del tejido, y la dependencia de tal 
efecto con la temperatura de almacenamiento.

El estrés hídrico de frutos de pepino (Cucumis sativus L.), almacenados a $62 \% \mathrm{HR}$ y $15.5^{\circ} \mathrm{C}$, afectó la firmeza e indujo el desarrollo de tejido esponjoso así como presencia de células colapsadas en el mesocarpio (Walter et al., 1990). Dichos cambios se relacionaron con la pérdida de adhesión intercelular y de turgencia por efecto de modificaciones en los componentes de la pared celular y citoplasma, respectivamente (Shackel et al., 1991; Waldron et al., 2003). Shibairo et al. (2002) reportaron que una pérdida de $20 \%$ de humedad en zanahoria (Daucus carota L. cv. 'Eagle') almacenada por $30 \mathrm{~d}$ a $13{ }^{\circ} \mathrm{C}$ y $79 \% \mathrm{HR}$, redujo en $63 \%$ el potencial hídrico y en $54 \%$ el potencial osmótico, con un deterioro considerable de la membrana celular del tejido. En toronja (Citrus paradisi Macf.) almacenada por $12 \mathrm{~d}$ a 20 ${ }^{\circ} \mathrm{C}$ y 45 \% HR, Alferez et al. (2010) observaron que ocurrió colapso celular en el albedo interno y el flavedo de los frutos, fenómeno representado por el cambio de valores positivos a negativos en el potencial de presión de dichos tejidos. En calabacita se desconoce si la pérdida de firmeza en esta hortaliza se debe a la reducción del potencial de presión o al incremento del potencial osmótico de los frutos inducido por la temperatura de almacenamiento.

El objetivo de este estudio fue conocer el efecto de la temperatura de almacenamiento sobre las variables hídricas, la integridad de la pared celular y la calidad de la calabacita tipo Zucchini durante postcosecha.

\section{MATERIALES Y MÉTODOS}

Material de estudio. Se obtuvieron frutos de calabacita tipo Zucchini $c v$. 'Raben' directamente de una empacadora ubicada en Navolato, Sinaloa, México ( $24^{\circ} 40^{\prime} 40^{\prime \prime} \mathrm{LN}, 107^{\circ}$ $47^{\prime} 38^{\prime \prime} \mathrm{LO}, 5 \mathrm{msnm}$ ). Los frutos cosechados en madurez hortícola ( $5 \mathrm{~cm}$ diámetro y $22 \mathrm{~cm}$ de longitud) se dividieron en dos lotes de 120 frutos cada uno; el primero se almacenó a $10{ }^{\circ} \mathrm{C}$ y el segundo a $20{ }^{\circ} \mathrm{C}$, ambos entre 85 y $90 \% \mathrm{HR}$. Cada tercer día se evaluó pérdida de peso, marchitamiento, contenido de agua (capacidad de retención de agua, potenciales hídrico, osmótico y de presión) y la anatomía del tejido.

Pérdida de peso. Se evaluó en 10 frutos con una balanza digital GF-2000 AND ${ }^{\circledR}$ (Japan). Para los cálculos se consideró el peso inicial y el peso final de los frutos, y los resultados se reportaron en porcentajes de pérdida acumulada de peso (Muy et al., 2004).

Marchitamiento. Se registró de manera subjetiva (visual) con base en la presencia de síntomas de deshidratación en la piel, en cinco frutos de calabacita, con una escala visual de marchitamiento del 1 al 5: 1 = nada, $2=$ ligero, 3
= moderado (pero todavía con calidad visual comercial), $4=$ moderadamente severo y $5=$ severo. Los valores $4 \mathrm{y}$ 5 correspondieron a frutos con marchitamiento excesivo, sin valor comercial, según lo recomendado por Cantwell y Thangaiah (2001).

Análisis de perfil de textura (TPA por sus siglas en inglés, texture profile analysis). El TPA se evaluó cada tercer día en cinco frutos con cáscara, por tratamiento. La determinación se hizo en la sección media del fruto con un penetrómetro DF GS 100 Chatillon ${ }^{\circledR}$ (USA), equipado con un disco de $5 \mathrm{~cm}$ de diámetro y adaptado a una base TCD200. La distancia de compresión se realizó con base en 10 \% del diámetro de cada fruto y la resistencia a la deformación se reportó como dureza $(\mathrm{N})$, gomosidad $\left(\mathrm{N} \mathrm{s}^{-1}\right)$, masticabilidad $\left(\mathrm{N} \mathrm{mm}^{-1}\right)$, cohesividad y elasticidad (adimensionales). La dureza es la fuerza máxima requerida para comprimir el fruto en el primer ciclo de presión; cohesividad es la relación del área bajo la curva de la segunda compresión dividida por el área bajo la curva de la primera compresión; gomosidad es el producto de la dureza por la cohesividad por tiempo; masticabilidad es el producto de la dureza por la cohesividad; elasticidad es la relación de la altura del tejido al final de la primera compresión dividida por la altura al inicio de la segunda compresión (Bourne, 2002).

Contenido relativo de agua (CRA). Se determinó por duplicado en tres frutos (Pomper y Breen, 1997), con modificaciones de Muy et al. (2004). Se cortaron discos de tejido de $20 \mathrm{~mm}$ de diámetro y $3 \mathrm{~mm}$ de espesor, con un sacabocados; se pesaron en una balanza analítica CP3245 Sartorius ${ }^{\circledR}$ (Germany), para obtener el peso fresco (Pf). Después, los discos se colocaron en agua destilada $\left(25 \pm 1^{\circ} \mathrm{C}\right)$ por $2 \mathrm{~h}$. La muestra se retiró del agua, se le eliminó el exceso de este líquido en la superficie y se determinó su peso a máxima turgencia $(P t)$. Finalmente, las muestras se secaron a $75^{\circ} \mathrm{C}$ por $48 \mathrm{~h}$ y se evaluaron para conocer el peso seco $(P s)$. El CRA se determinó con la ecuación:

$$
C R A(\%)=\left[(P f-P s)(P t-P s)^{-1}\right] 100
$$

Potenciales hídrico $\left(\Psi_{w}\right)$, osmótico $\left(\Psi_{s}\right)$ y de presión $\left(\Psi_{p}\right)$. Para el $\Psi_{w}$ se utilizó el método de volumen constante (Shibairo et al., 1997). Se cortaron trozos del fruto de 40 x $5 \mathrm{~mm}$ a lo largo y ancho del pericarpio de la calabacita, los cuales se pesaron y colocaron en cuatro soluciones de sacarosa con distinta concentración $(0.2,0.3,0.4$ y $0.5 \mathrm{M})$. A estas soluciones se les determinó el potencial osmótico mediante la ecuación $\Psi s=-C i R T$, donde $C i$ es la concentración molal de la sacarosa, $R=$ constante ideal de los gases $\left(0.0083 \mathrm{~kg} \mathrm{MPa} \mathrm{mol}^{-1} \mathrm{~K}^{-1}\right)$ y $T=$ temperatura (K). En una solución el $\Psi_{p}$ es igual a cero, y por tanto $\Psi_{w}=\Psi_{s}$. Las muestras se dejaron $2 \mathrm{~h}$ en reposo, se retiraron de la solución, se les eliminó el agua excedente de la superficie y se registró 
el peso. Posteriormente, se graficó el valor del $\Psi_{w}$ contra el peso ganado o perdido. La concentración de la sacarosa que no causó variación en el peso de la muestra, se consideró equivalente al potencial hídrico (Salisbury y Ross, 2000).

El $\Psi_{s}$ se midió con un osmómetro de presión de vapor 5520 Wescor ${ }^{\circledR}$ (USA), de acuerdo con la metodología propuesta por Turner (1981) modificada por Muy et al. (2004). Se agregaron $10 \mu \mathrm{L}$ de savia del fruto en un disco de papel filtro de $0.32 \mathrm{~cm}^{2}$ y se colocó en la cámara de recepción de la muestra. La savia se obtuvo de trozos de tejidos congelados a $-20^{\circ} \mathrm{C}$, mismos que se descongelaron a temperatura ambiente para romper las membranas celulares y así llevar el potencial de turgencia a cero. La calibración del equipo se hizo con soluciones de potencial osmótico de 290, 1000 y $100 \mathrm{mmol} \mathrm{kg}^{-1}$ de $\mathrm{NaCl}$ (en este orden de calibración). Los resultados fueron en $\mathrm{mmol} \mathrm{\textrm {kg } ^ { - 1 }}$. La conversión de molalidad a potencial osmótico se obtuvo con la ecuación de Van't Hoff: $(\Psi s)=-C i R T$, (Salisbury y Ross, 2000). El $\Psi_{\mathrm{p}}$ se calculó por diferencia entre el potencial hídrico y el osmótico $\left(\Psi_{p}=\Psi_{w}-\Psi_{s}\right)$ según Turner (1981). Estas variables se analizaron cada $3 \mathrm{~d}$ en los frutos almacenados por $9 \mathrm{~d}$ a 10 y $20^{\circ} \mathrm{C}$.

Análisis histológico. La fijación del tejido de calabacita se hizo en trozos de aproximadamente $1 \mathrm{~cm}^{3}$ del pericarpio del fruto. Se fijaron muestras de la parte del pericarpio (epicarpio, mesocarpio y endocarpio) del fruto. Las muestras se colocaron en solución fijadora FAA, constituida por $10 \%$ de formaldehido a $37 \%, 50 \%$ de etanol a $96 \%, 5 \%$ de ácido acético glacial y $35 \%$ de agua. A continuación las muestras se lavaron con agua corriente, se deshidrataron en alcoholes graduados y xileno, y se infiltraron en parafina. Posteriormente, se hicieron cortes del tejido de calabacita incluido en parafina a $10 \mu \mathrm{m}$ de grosor con un micrómetro rotatorio RM2125 Leyca ${ }^{\circledR}$ (Germany). Los cortes se montaron sobre un portaobjetos con adhesivo Haupt (1 \% gelatina, $13 \%$ glicerol, $2 \%$ fenol y agua), se tiñeron con safranina y verde fijo, y se cubrieron con un portaobjeto adherido con bálsamo de Canadá para obtener preparaciones permanentes (Osuna et al., 2008). Los cortes anatómicos fueron observados en un microscopio óptico 1122-100 Zeizz ${ }^{\circledR}$ Axiostar (México). En el análisis se dio énfasis al deterioro celular de acuerdo con la ubicación del tejido en el epicarpio, mesocarpio y endocarpio del fruto.

Análisis estadístico. Los datos de las variables $\Psi_{w^{\prime}} \Psi_{s}, \Psi_{p}$ y CRA se analizaron mediante un diseño completamente al azar con arreglo de dos factores (temperatura y tiempo). En las pruebas para analizar la variable pérdida de peso se utilizó un diseño de medidas repetidas, con un factor anidado (temperatura) y un factor cruzado (tiempo). Cuando el análisis de varianza resultó significativo $(\mathrm{P} \leq 0.05)$, se hizo comparación de medias por Tukey con $\alpha=0.05$. Se utilizó el paquete estadístico MINITAB versión 14 (MINITAB, 2008).

\section{RESULTADOS Y DISCUSIÓN}

\section{Pérdida de peso}

El tiempo y la temperatura de almacenamiento afectaron $(\mathrm{P} \leq 0.05)$ la pérdida de peso de los frutos de calabacita $(\mathrm{Fi}-$ gura 1). Los almacenados a 10 y $20^{\circ} \mathrm{C}$ perdieron en promedio 0.94 y $1.34 \%$ de peso $\mathrm{d}^{-1}$, respectivamente; la pérdida de peso fue diferente después de $3 \mathrm{~d}$ de almacenamiento. Las calabacitas presentaron marchitamiento moderadamente severo (valores de marchitez $\geq 4$ ), sin valor comercial a los 3 y $6 \mathrm{~d}$ de almacenamiento a 20 y $10{ }^{\circ} \mathrm{C}$, respectivamente, lo cual se relacionó con una pérdida acumulada de peso de $6 \%$ y una reducción de $11 \%$ en CRA (Figura 1). Lo anterior sugiere que la pérdida de calidad por marchitez de la calabacita se puede acelerar o retrasar en función a las condiciones de almacenamiento postcosecha, y la marchitez inicia con $6 \%$ de pérdida de peso, lo que coincide con Sargent y Maynard (2002) quienes reportaron pérdida de calidad en cucurbitáceas con este mismo porcentaje de pérdida de peso.

En un estudio con calabacita Zucchini cv. 'Elite' cosechada a dos semanas de desarrollo (200 g de peso aproximadamente) y sin síntomas de daño mecánico, los frutos mostraron una pérdida de peso de $11 \%$ durante el almacenamiento por $12 \mathrm{~d}$ a $20^{\circ} \mathrm{C}$ y $85 \% \mathrm{HR}$ (Serrano et al., 1998), la cual es menor a la obtenida en este estudio (16\%) bajo las mismas condiciones. Ben-Yehoshua (1987) reportó que las habas verdes (Vicia faba L.), zarzamora (Rubus fructicosus L.) y fresa (Fragaria vesca L.) pierden su frescura cuando alcanzan de 3 a $10 \%$ de pérdida de peso, y presentan pérdida de la calidad visual (marchitez) cuando la pérdida de peso es mayor de $6 \%$, tal como se observó en el presente estudio. Lechuga (Lactuca sativa L.) y espinaca (Spinacia oleracea L.) son productos vegetales más susceptibles a la deshidratación y su calidad se deteriora con tan solo $3 \%$ de pérdida de peso, en tanto que el repollo (Brassica oleracea L.) tolera hasta $10 \%$. La pérdida de calidad por marchitez del pepino (Cucumis sativus) de mesa se presentó con una pérdida de $6 \%$ del peso, pero es posible retrasar la aparición del síntoma mediante el almacenamiento con condiciones de humedad relativa mayor a $95 \%$ y con el uso de una película comestible que reduce significativamente la velocidad de transpiración en los frutos (Muy et al., 2004). Pérdidas similares se observaron aquí en las calabacitas por efecto de la temperatura de almacenamiento, pues presentaron mayor marchitez y pérdida de calidad a mayor temperatura.

\section{Análisis de perfil de textura (TPA)}

Las calabacitas al momento del corte mostraron una dureza 


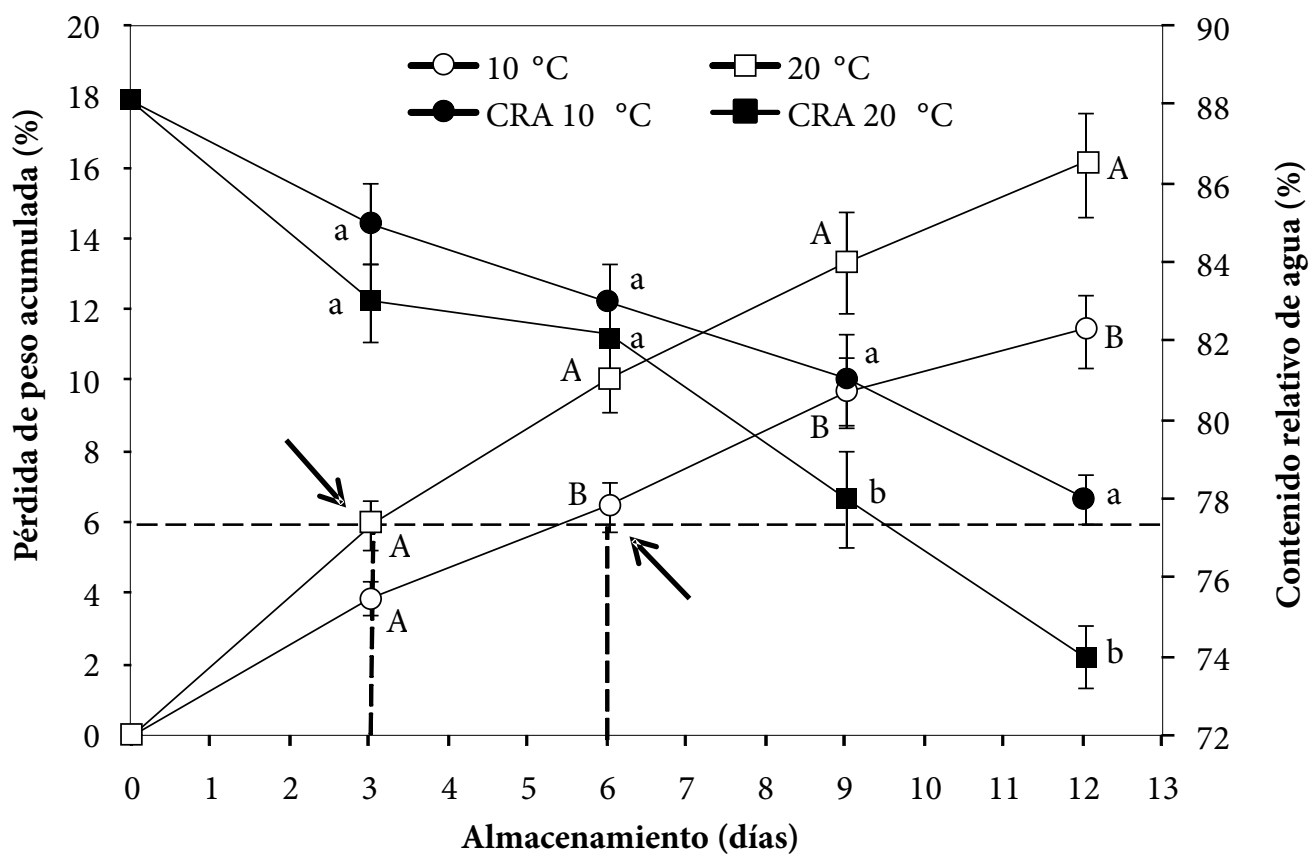

Figura 1. Pérdida de peso $(\circ, \square)$ y contenido relativo de agua $(C R A \bullet, \bullet)$ en frutos de calabacita Zucchini almacenados a 10 y $20^{\circ} \mathrm{C}$. Las barras verticales en cada punto son el error estándar de 10 frutos. Las flechas indican el tiempo donde se observaron los primeros sintomas de marchitez en los frutos. Líneas discontinuas representan el tiempo y porcentaje de pérdida de peso al momento de la marchitez del fruto. Mismas letras entre condiciones de almacenamiento indican promedios estadísticamente iguales, por variable y por día $(\mathrm{P}<0.05)$.

de $117 \mathrm{~N}$ y durante los $12 \mathrm{~d}$ en el almacenamiento a 10 y $20^{\circ} \mathrm{C}$ la dureza del fruto disminuyó en 88 y $98 \%$, respectivamente (Cuadro 1). La mayor pérdida de dureza ocurrió después de $3 \mathrm{~d}$ de almacenamiento, sin diferencia $(\mathrm{P}>0.05)$ con los frutos almacenados hasta por $9 \mathrm{~d}$ a 10 y $20^{\circ} \mathrm{C}$. A los $12 \mathrm{~d}$ de almacenamiento la dureza y la masticabilidad de las calabazas resultaron significativamente $(\mathrm{P} \leq 0.5)$ mayores en los frutos a $10{ }^{\circ} \mathrm{C}$ que en los almacenados a $20^{\circ} \mathrm{C}$, lo que según Bourne (2002) indica una mayor resistencia a la disgregación del tejido del fruto.

La masticabilidad, cohesividad y elasticidad de los frutos no presentaron variaciones significativas por efecto de la temperatura, a diferencia de lo observado al final del almacenamiento (Cuadro 1). Estas variables de textura son indicadores del trabajo y el tiempo requerido para la disgregación del tejido durante la masticación (Bourne, 2002).

La presencia de marchitez a los 3 y $6 \mathrm{~d}$ de almacenamiento de los frutos no se relacionó con las variables del perfil de textura de las calabacitas, debido posiblemente a que la marchitez se observa en el epicarpio del fruto y la textura del tejido se analiza considerando todas sus partes (epicarpio, mesocarpio y endocarpio).

En calabaza 'Delica' (C. maxima), Sunil et al. (1999) observaron que la pérdida de consistencia del tejido se asocia con cambios en la dureza ( 60 a $44 \mathrm{~N}$ ) y en la gomosidad ( 10 a $8 \mathrm{~N}$ ), sin cambios considerables en elasticidad (4), masticabilidad $\left(40 \mathrm{~N} \mathrm{~mm}^{-1}\right)$ y cohesividad $(0.17 \mathrm{~mm})$ del fruto durante tres meses de almacenamiento. Esto indica que la integridad celular y la forma de disgregación del tejido de las cucurbitáceas (Figura 3) están relacionadas con la elasticidad y resistencia de las células, y no con el contenido de agua.

\section{Contenido relativo de agua (CRA)}

Después de $6 \mathrm{~d}$ de almacenamiento, los frutos a $20^{\circ} \mathrm{C}$ presentaron una mayor reducción del $C R A(\mathrm{P} \leq 0.05)$ que los frutos que permanecieron a $10^{\circ} \mathrm{C}$ (Figura 1). Bajo estas condiciones, a los $12 \mathrm{~d}$ las calabacitas a 20 y $10^{\circ} \mathrm{C}$ perdieron alrededor de 16 y $11 \%$ del CRA, respectivamente, disminución que se relacionó con el tiempo y la temperatura de almacenamiento. Cuando las calabacitas mostraron síntomas de marchitez moderadamente severo $(6 \%$ de pérdida de peso), éstas presentaron $83 \%$ de CRA. Sin embargo, las calabacitas almacenadas durante 6 y $9 \mathrm{~d}$ a 20 y $10{ }^{\circ} \mathrm{C}$, presentaron un cambio de valores positivos a valores de cero o negativos en el potencial de presión del tejido, lo cual se asoció con plasmólisis celular, que según Alferez et al. (2010) ocurre con un CRA entre 81 y $82 \%$ y una pérdida de peso acumulada superior a $10 \%$. 
Cuadro 1. Análisis de perfil de textura en frutos de calabacita zucchini durante el almacenamiento a 10 y $20^{\circ} \mathrm{C}$.

\begin{tabular}{lcccccc}
\hline Variable & $\begin{array}{c}\text { Temperatura } \\
\left({ }^{\circ} \mathrm{C}\right)\end{array}$ & \multicolumn{5}{c}{ Almacenamiento $(\mathrm{d})$} \\
\cline { 3 - 7 } & 10 & 0 & 3 & 6 & 9 & 12 \\
\hline Dureza $(\mathrm{N})$ & 20 & $117.1 \pm 35.1$ & $52.9 \mathrm{a} \pm 13.1$ & $50.4 \mathrm{a} \pm 8.6$ & $64.4 \mathrm{a} \pm 16.3$ & $13.1 \mathrm{a} \pm 1.7$ \\
& 10 & $64.5 \mathrm{a} \pm 16.1$ & $57.2 \mathrm{a} \pm 24.5$ & $59.1 \mathrm{a} \pm 24.1$ & $2.2 \mathrm{~b} \pm 1.4$ \\
Gomosidad $\left(\mathrm{N} \mathrm{s}^{-1}\right)$ & 20 & $39.5 \pm 9.7$ & $24.1 \mathrm{a} \pm 4.5$ & $21.1 \mathrm{a} \pm 5.3$ & $27.2 \mathrm{a} \pm 7.4$ & $8.7 \mathrm{a} \pm 0.2$ \\
& 10 & $25.3 \mathrm{a} \pm 4.1$ & $23.5 \mathrm{a} \pm 10.2$ & $23.1 \mathrm{a} \pm 8.2$ & $-0.1 \mathrm{~b} \pm 0.5$ \\
Masticabilidad $\left(\mathrm{N} \mathrm{mm}^{-1}\right)$ & 20 & $126.8 \pm 37.1$ & $89.2 \mathrm{a} \pm 20.1$ & $69.1 \mathrm{a} \pm 24.6$ & $89.2 \mathrm{a} \pm 38.7$ & $12.7 \mathrm{a} \pm 10.7$ \\
& 10 & & $92.1 \mathrm{a} \pm 23.7$ & $82.0 \mathrm{a} \pm 39.9$ & $80.5 \mathrm{a} \pm 32.9$ & $0.0 \mathrm{~b} \pm 0.1$ \\
Cohesividad $(\mathrm{mm})$ & 20 & $0.3 \pm 0.0$ & $0.4 \mathrm{a} \pm 0.0$ & $0.4 \mathrm{a} \pm 0.1$ & $0.4 \mathrm{a} \pm 0.1$ & $0.4 \mathrm{a} \pm 0.3$ \\
& 10 & & $0.4 \mathrm{a} \pm 0.1$ & $0.4 \mathrm{a} \pm 0.1$ & $0.4 \mathrm{a} \pm 0.0$ & $-0.1 \mathrm{a} \pm 0.2$ \\
Elasticidad $(\mathrm{mm})$ & 20 & $3.2 \pm 0.4$ & $3.4 \mathrm{a} \pm 0.2$ & $3.2 \mathrm{a} \pm 0.4$ & $3.1 \mathrm{a} \pm 0.6$ & $1.2 \mathrm{a} \pm 0.7$ \\
& & & $3.6 \mathrm{a} \pm 0.4$ & $3.4 \mathrm{a} \pm 0.9$ & $3.4 \mathrm{a} \pm 0.5$ & $0.3 \mathrm{a} \pm 0.2$ \\
\hline
\end{tabular}

Media y desviación estándar de 10 repeticiones. Letras iguales en na hilera señalan promedios estadísticamente iguales entre sí ( $\mathrm{P}>0.05)$.

Las vainas frescas de okra (Albelmoschus esculentus) cv. 'Amarelinho' almacenadas por $4 \mathrm{~d}$ a 10 y $25^{\circ} \mathrm{C}$ presentaron pérdidas excesivas de agua de 14 y $23 \%$ y valores de CRA de 74 y $71 \%$, respectivamente (Finger et al., 2008), por lo que se requiere utilizar alguna película comestible que recubra al fruto y reduzca la velocidad de transpiración del fruto. A pesar de la delgada cutícula que tiene la calabacita al cosecharse como fruto inmaduro, esta hortaliza no mostró valores de CRA tan bajos como la okra, pero fueron similares al del pepino 'Pickle' con 92 a $77 \%$ de CRA durante el almacenamiento por $12 \mathrm{~d}$ a $93 \% \mathrm{HR}$ (Walter et al., 1990), e inferior a los valores reportados en pepino de mesa con CRA de 91 a $88 \%$ a los $12 \mathrm{~d}$ a $24{ }^{\circ} \mathrm{C}$ (Muy et al., 2004), ambos con desarrollo de tejido esponjoso durante el almacenamiento.

\section{Potenciales hídrico $\left(\Psi_{w}\right)$, osmótico $\left(\Psi_{s}\right)$ y de presión $\left(\Psi_{p}\right)$}

Al inicio del estudio, el $\Psi_{w}$ de las calabacitas fue de -0.72 MPa y a los $9 \mathrm{~d}$ de almacenamiento a 10 y $20^{\circ} \mathrm{C}$ el potencial se redujo a -0.79 y $-0.86 \mathrm{MPa}$, respectivamente (Figura 2), descenso que fue significativo $(\mathrm{P} \leq 0.05)$. Valores inferiores de $\Psi_{w}$ corresponden a calabacitas almacenadas a $9 \mathrm{~d}$ a $20^{\circ} \mathrm{C}$, las cuales presentaron la mayor pérdida de peso con $13.5 \%$ (Figura 1). Así mismo, los cambios en los potenciales $\left(\Psi_{w^{\prime}}\right.$ $\Psi_{s}$ y $\left.\Psi_{p}\right)$ de los frutos almacenados a $20^{\circ} \mathrm{C}$ se asociaron con la histología del tejido (Figura 3), en los que se observó mayor desorganización celular que en los almacenados a $10^{\circ} \mathrm{C}$.

Durante el almacenamiento de los frutos por $6 \mathrm{~d}$ a $20^{\circ} \mathrm{C} \mathrm{y}$ $9 \mathrm{~d}$ a $10^{\circ} \mathrm{C}$, el $\Psi_{s}$ de las calabacitas fue ligeramente menor al $\Psi_{w}$, debido posiblemente al incremento en la concentración de sólidos solubles en las células del tejido causado por la degradación de macromoléculas durante la actividad me- tabólica en postcosecha. Tal respuesta también puede ser atribuida a un aumento en la concentración de solutos por efecto de la pérdida de agua del fruto. En relación con lo anterior, el $\Psi_{p}$ presentó una disminución significativa $(\mathrm{P} \leq$ 0.05 ) durante el almacenamiento de los frutos, pues bajó de $0.5 \mathrm{MPa}$ a valores de cero o negativos a los 6 y $9 \mathrm{~d}$ a 20 y 10 ${ }^{\circ} \mathrm{C}$, respectivamente. Los $\Psi_{p}$ iguales a cero coinciden con la intersección de las líneas de los $\Psi_{w}$ y $\Psi_{s}$ (Figura 2); dicha condición fisiológica indica plasmólisis celular del tejido de los frutos, según Salisbury y Ross (2000). Durante este fenómeno se observó mayor pérdida de la integridad celular y la aparición de espacios intracelulares, en comparación con los tejidos que no habían alcanzado ese cambio (Figura 3). La plasmólisis celular de la calabacita se presentó después de una marchitez severa del fruto, con pérdida total de la calidad.

En flor de calabaza, Villalta et al. (2004) reportaron colapso celular durante el almacenamiento por $14 \mathrm{~d}$ a $5{ }^{\circ} \mathrm{C}$, acompañado de pérdida total de la calidad comercial y el desarrollo de tejido necrótico. En zanahoria (Daucus carota L.) cv. 'Eagle', Shibairo et al. (1997) observaron un efecto negativo en la apariencia del fruto durante el almacenamiento, lo cual se relacionó con una reducción del $\Psi_{\mathrm{s}}$ de -1.3 a $-1.7 \mathrm{MPa}$ a los 7 y 21 a $13{ }^{\circ} \mathrm{C}$, respectivamente, debido a la pérdida de agua del tejido. En frutos de kiwi 'Hayward' (Actinidia deliciosa) los cambios en $\Psi_{w}$ fueron de -1.02 a -1.7 y de -1.08 a -1.6 en $\Psi_{s}$, y de $+0.06 \mathrm{MPa}$ a valores negativos en $\Psi_{p}$, con un CRA de 95 a $90 \%$, del día cero a los $14 \mathrm{~d}$ a 20 ${ }^{\circ} \mathrm{C}$, en donde el inicio de la plasmólisis celular fue a los $14 \mathrm{~d}$ (Burdon y Clark, 2001), contra 6 y 9 d en calabacitas a 20 y $10{ }^{\circ} \mathrm{C}$, respectivamente. 

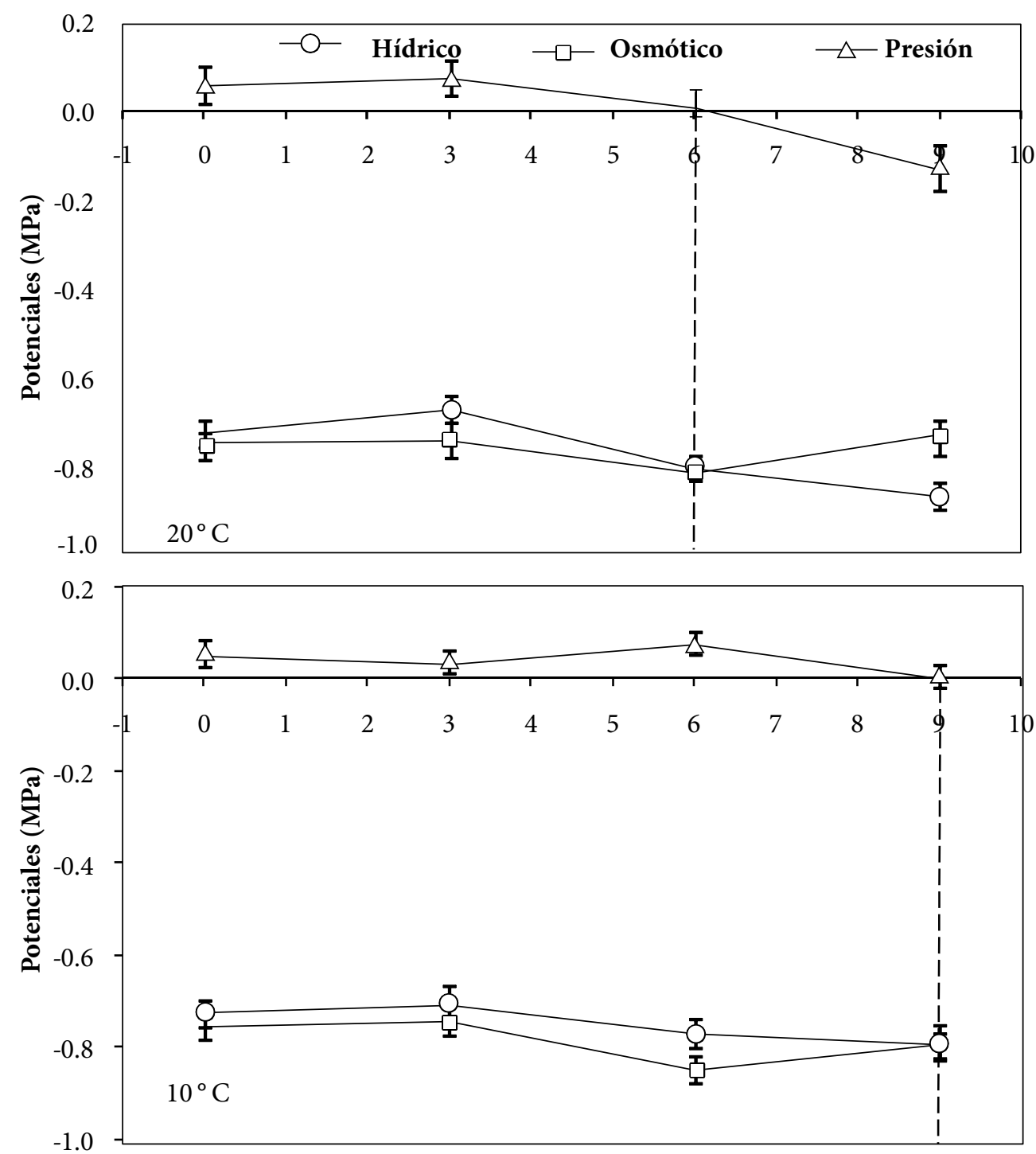

Almacenamiento (días)

Figura 2. Potenciales hídrico, osmótico y de presión en frutos de calabacita Zucchini almacenados a 20 y $10^{\circ} \mathrm{C}$. Línea vertical discontinua muestra el tiempo donde se presentó el cambio del potencial de presión de valores positivos a negativos, indicativo de plasmólisis celular. Las barras verticales en cada punto son el error estándar de tres frutos.

\section{Análisis histológico}

La temperatura y el tiempo de almacenamiento afectaron la integridad celular de los tejidos de calabacita (Figura 3). Al día cero el epicarpio del fruto presentó células compactas sin deterioro (Figura 3-1), totalmente hidratadas y turgentes $\left(\Psi_{p}=0.6 \mathrm{MPa}\right.$, Figura 2); mientras que en las células del mesocarpio se apreciaron espacios vacíos (Figura 3-2), los cuales se incrementaron en el endocarpio (Figura 3-3).

A los $12 \mathrm{~d}$ de almacenamiento a $10^{\circ} \mathrm{C}$, las células del epicarpio no mostraron cambios considerables con relación al día cero, ni pérdida de integridad celular (Figura 3-1A).
Esto podría explicar el por qué no coincide el tiempo de marchitez de la fruta con la plasmólisis celular, ya que la desintegración celular inició en el interior y luego avanzó hacia al exterior del pericarpio. En los frutos almacenados $12 \mathrm{~d}$ a $20^{\circ} \mathrm{C}$ se detectó desintegración celular desde el epicarpio (Figura 3-1B). En ambas temperaturas de almacenamiento se observó que en las células del mesocarpio hubo un incremento gradual del aerénquima (Figura $3-2 \mathrm{~A}, 2 \mathrm{~B})$, con presencia de tejido colapsado en la sección del endocarpio (3-3A, 3B), con mayor deterioro en los tejidos a $20^{\circ} \mathrm{C}$ (Figura 3-3B). Bajo estas dos condiciones $\left(12 \mathrm{~d}\right.$ a 10 y $\left.20^{\circ} \mathrm{C}\right)$, los frutos alcanzaron una pérdida de peso mayor a $6 \%$ (Figura 1), presentaron síntomas 

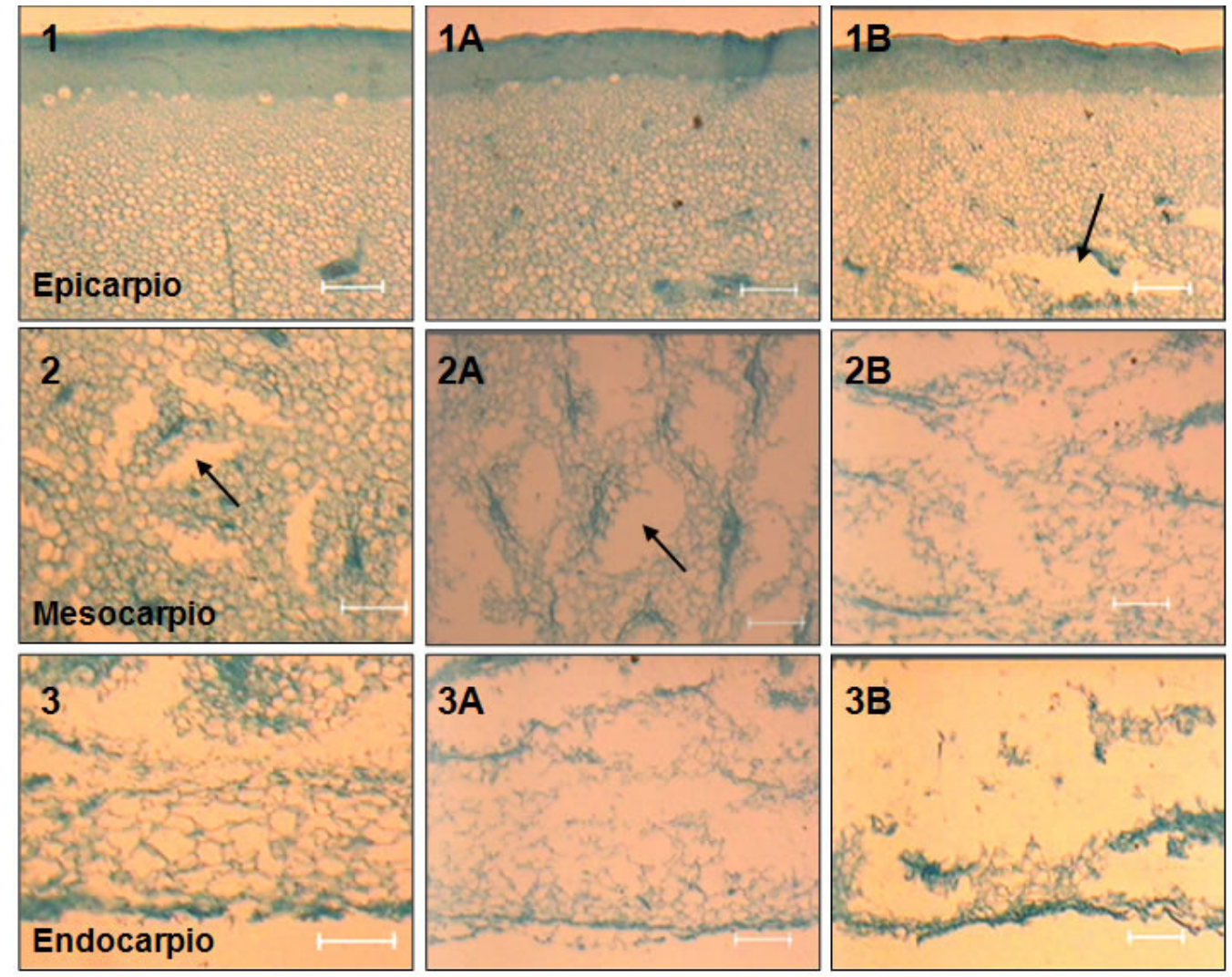

Figura 3. Integridad del tejido de frutos de calabacita durante el almacenamiento. 1 = Epicarpio; 2 = Mesocarpio; 3 = Endocarpio, al día inicial. A = Frutos almacenados por $12 \mathrm{~d} \mathrm{a} 10^{\circ} \mathrm{C} . \mathrm{B}$ $=$ Frutos almacenados por $12 \mathrm{~d}$ a $20^{\circ} \mathrm{C}$. Barra blanca de referencia $=200 \mu \mathrm{m}$. Flechas indican espacios vacíos.

de marchitez, $\Psi_{p}<0 \mathrm{MPa}$ y plasmólisis celular. A pesar de presentar desintegración celular en el mesocarpio y endocarpio, la calabacita almacenada por $12 \mathrm{~d}$ a $10{ }^{\circ} \mathrm{C}$ mostró menor pérdida de peso y mayor $C R A$ en comparación con las almacenadas a $20^{\circ} \mathrm{C}$.

Walter et al. (1990) reportaron que el fruto de pepino var. 'Pickle' almacenado a $15^{\circ} \mathrm{C}$ con $93 \%$ HR mostró daño en las células del parénquima debido a la pérdida de agua y a daños en la estructura de los componentes de la pared celular. Después de $9 \mathrm{~d}$ de almacenamiento, el fruto alcanzó un CRA menor a $85 \%$ y desarrolló tejido esponjoso, con células plasmolizadas que afectaron la integridad del tejido, similar a lo observado en calabacita a $20^{\circ} \mathrm{C}$. En champiñones (Agaricus bisporus), Zivanovic et al. (2000) también registraron pérdida de turgencia y contenido del polisacárido quitina en las células del tejido durante el almacenamiento por $9 \mathrm{~d} \mathrm{a} 12^{\circ} \mathrm{C}$ y $95 \% \mathrm{HR}$; el daño se relacionó con espacios intracelulares en la superficie del píleo del hongo, células colapsadas en las hifas y pérdida de integridad de la vacuola. En frutos de pérsimo (Diospyros kaki L.) se obser- vó pérdida de integridad de las paredes celulares y adhesión entre células cuando los frutos se almacenaron por $20 \mathrm{~d}$ a 15 ${ }^{\circ} \mathrm{C}$ (Salvadora et al., 2008), similar a las características aquí observadas en el mesocarpio y endocarpio de las células de calabacita durante el almacenamiento a 10 y $20^{\circ} \mathrm{C}$.

\section{CONCLUSIONES}

Una pérdida de peso de $6 \%$ en calabacita Zucchini se relacionó con los primeros síntomas de marchitez y pérdida de calidad comercial de los frutos. La temperatura de almacenamiento de $10^{\circ} \mathrm{C}$ retrasó significativamente la pérdida de agua y la aparición de plasmólisis celular de los tejidos, en comparación con los frutos almacenados a $20^{\circ} \mathrm{C}$. No se encontró una relación entre el tiempo del desarrollo de la marchitez de la calabaza y la plasmólisis del tejido. La plasmólisis celular de la calabacita se presentó cuando el contenido relativo de agua en los frutos se redujo a valores entre 81 a $82 \%$, con una pérdida de peso mayor a $10 \%$. Los principales cambios histológicos en el pericarpio del fruto se desarrollaron del endocarpio hacia el epicarpio, que consistieron en una mayor 
presencia de células colapsadas y pérdida de adhesión celular en los frutos almacenados a $20^{\circ} \mathrm{C}$.

\section{AGRADECIMIENTOS}

Al apoyo técnico de la M.C. Laura Aracely Contreras Angulo, la M.C. Rosabel Vélez de la Rocha y la Ing. Rosalba Contreras Martínez. Así como al Consejo Nacional de Ciencia y Tecnología, por el apoyo económico otorgado a la Ing. Vania Urias Orona.

\section{BIBLIOGRAFÍA}

Alferez F, B Alquezar, J Burns, L Zacarias (2010) Variation in water, osmotic and turgor potential in peel of 'Marsh' grapefruit during development of postharvest peel pitting. Postharv. Biol. Technol. 56:44-49.

Balandrán-Quintana R R, A M Mendoza-Wilson, A Gardea-Béjar, I Vargas-Arispuro, M A Martínez-Téllez (2003) Irreversibility of chilling injury in zucchini squash (Cucurbita pepo L.) could be a programmed event long before the visible symptoms are evident. Biochem. Biophys. Res. Comm. 307:553-557.

Ben-Yehoshua S (1987) Transpiration, water stress, and gas exchange. In: Postharvest Physiology of Vegetables. J Weichmann (ed). Marcel Dekker, Inc. New York. USA. pp:113-170.

Brew B S, A D Berry, S A Sargent, N I Shaw, D J Cantliffe (2006) Determination of optimum storage conditions for 'baby' summer squash fruit (Cucurbita pepo). Proc. Florida State Hort. Soc. 119:343-346.

Bourne M (2002) Food Texture and Viscosity: Concept and Measurement. Academic Press. USA. 182 p.

Burdon J, C Clark (2001) Effect of postharvest water loss on 'Hayward' Kiwifruit water status. Postharv. Biol. Technol. 22:215-225.

Cantwell M, A Thangaiah (2001) Delays to cool affect visual quality, firmness and gloss of bell peppers and eggplants. Perishables Handling Quarterly. University of California. Issue $\mathrm{N}^{\circ} 107$. pp:17-20.

Carvajal F, C Martinez, M Jamilena, D Garrido (2011) Differential response of zucchini varieties to low storage temperature. Sci. Hort. 130:90-96.

Finger F L, M Della-Justina, V Dias, M Puiatti (2008) Temperature and modified atmosphere affect the quality of okra. Sci. Agric. (Brazil) 65:360-364.

MINITAB (2008) Statically software. Ver. 14.1. Disponible en: http:// www.minitab.com

Mohammed M, J K Brecht (2003) Immature fruit vegetables. In: Postharvest Physiology and Pathology of Vegetables. A Bartz Jerry y K Brecht Jeffery (eds). Marcel Dekker, Inc. University of Florida Gainesville, Florida, USA. pp:241-256.

Muy R D, J Siller-Cepeda, J Díaz-Pérez, B Valdez-Torres (2004) Efecto de las condiciones de almacenamiento y el encerado en el estado hídrico y la calidad poscosecha de pepino de mesa. Rev. Fitotec. Mex. 27:157-165.
Osuna E T, R G Valenzuela, R D Muy, B A Gardea, R M Villareal (2008) Expresión del sexo y anatomía floral del litchi (Litchi chinensis Sonn.). Rev. Fitotec. Mex. 31:51-56.

Paull R E (1999) Effect of temperature and relative humidity on fresh commodity quality. Postharv. Biol. Technol. 15:263-277.

Pomper K W, P J Breen (1997) Expansion and osmotic adjustment of strawberry fruit during water stress. J. Amer. Soc. Hort. Sci. 122:183-189.

Salisbury F B, C W Ross (2000) Osmosis. In: Fisiología de las Plantas. F B Salisbury, C W Ross (eds). Thomson Learning. España. pp:65-93.

Salvadora A, L Arnala, C Besadaa, V Larreab, I Hernandoc, I PérezMunuerac (2008) Reduced effectiveness of the treatment for removing astringency in persimmon fruit when stored at $15^{\circ} \mathrm{C}$ physiological and microstructural study. Postharv. Biol. Technol. 49:340-347.

Sargent A S, D N Maynard (2002) Cucurbits. In: Crop Post-harvest: Science and Technology. P Golob (ed). Perishables 3. John Wiley \& Sons. England. pp:286-326.

Serrano M, M Pretel, M Martínez-Madrid, F Félix, F Riquelme (1998) $\mathrm{CO}_{2}$ treatment of zucchini squash reduces chilling-induced physiological changes. J. Agric. Food Chem. 46:2465-2468.

Shackel K A, C Greve, J M Labavitch, H Ahmadi (1991) Cell turgor changes associated with ripening in tomato pericarp tissue. Plant Physiol. 97:814-816.

Shibairo S I, M K Upadhyaya, P M Toivonen (1997) Postharvest moisture loss characteristics of carrot (Daucus carota L.) cultivars during short-term storage. Sci. Hort. 71:1-12.

Shibairo S I, M K Upadhyaya, P M Toivonen (2002) Changes in water potential, osmotic potential, a tissue electrolyte leakage during mass loss in carrots stored under different conditions. Sci. Hort. 95:13-21.

SIAP, Servicio de Información Agroalimentaria y Pesquera (2012) Producción agrícola de calabacita, 2010. Disponible en: http:// www.siap.gob.mx/index.php?option $=\mathrm{com}_{-}$wrapper\&view $=\mathrm{w}$ rapper\&Itemid $=351$. (Marzo 2012).

Sunil R R, P L Hurst, L D Melton (1999) Texture and the cell wall polysaccharides of buttercup squash 'Delica' (Cucurbita maxima). New Zeal. J. Crop Hort. Sci. 27:133-143.

Suslow T, M Cantwell (2009) Squash (soft rind). Postharvest Technology. Research Information Center. Produce/ProduceFacts/ veg/squash.shtml. Department of Plant Sciences. University of California. pp:1-4.

Turner N C (1981) Techniques and experimental approaches for the measurement of plant water status. Plant Soil 58:339-366.

Villalta A M, M Ergun, A D Berry, N Shaw, S A Sargent (2004) Quality changes of yellow summer squash blossoms (Cucurbita pepo) during storage. Acta Hort. 659:831-834.

Waldron K W, M L Parker, A C Smith (2003) Plant cell walls and food quality. Food Sci. Food Safety 2:101-1149.

Walter W M, D G Epley, R F McFeeters (1990) Effect of water stress on stored pickling cucumbers. J. Agric. Food Chem. 38:2185-2191.

Zivanovic S, R W Buescher, K S Kim (2000) Textural changes in mushrooms (Agaricus bisporus) associated with tissue ultrastructure and composition. J. Food Sci. 65:1404-1408. 